\title{
Computation of Worst Operation Scenarios Under Uncertainty for Static Security Management
}

Florin Capitanescu and Louis Wehenkel

\begin{abstract}
This paper deals with day-ahead static security assessment with respect to a postulated set of contingencies while taking into account uncertainties about the next day system conditions. We propose a heuristic approach to compute the worst-case under operation uncertainty for a contingency with respect to overloads. We formulate this problem as a non-convex nonlinear bilevel program that we solve approximately by a heuristic approach which relies on the solution of successive optimal power flow (OPF) and security-constrained optimal power flow (SCOPF) problems of a special type. The method aims at revealing those combinations of uncertainties and contingencies for which the best combination of preventive and corrective actions would not suffice to ensure security. Extensive numerical results on a small, a medium, and a very large system prove the interest of the approach.
\end{abstract}

Index Terms-Bilevel programming, operation under uncertainty, optimal power flow, security-constrained optimal power flow, worst-case analysis.

\section{NOMENCLATURE}

In the paper vectors are written using bold characters.

\section{A. Sets}

$\begin{array}{ll}\mathbf{g}_{0} & \begin{array}{l}\text { Set of power flow equations in pre-contingency } \\ \text { state. }\end{array} \\ \mathbf{g}_{c} & \text { Set of power flow equations after contingency } c . \\ \mathbf{h}_{0} & \text { Set of operating limits in pre-contingency state. } \\ \mathbf{h}_{c} & \text { Set of operating limits after contingency } c . \\ \mathcal{K} & \text { Set of postulated contingencies. } \\ \mathcal{V C} & \text { Set of violated constraints. } \\ \mathcal{P C} & \text { Set of problematic constraints. } \\ \mathcal{A P C} & \text { Union of all sets of problematic constraints. } \\ \mathcal{P P} & \text { Set of problematic patterns. }\end{array}$

Manuscript received May 04, 2012; revised August 14, 2012; accepted September 19, 2012. The work of F. Capitanescu was supported by the National Research Fund Luxembourg in the frame of the CORE project "Reliable and efficient distributed electricity generation in smart grids", C11/SR/1278568. The work of L. Wehenkel was supported by the Belgian Network DYSCO (Dynamical Systems, Control, and Optimization), funded by the Interuniversity Attraction Poles Programme, initiated by the Belgian State, Science Policy Office. The scientific responsibility rests with the authors. Paper no. TPWRS-00378-2012.

F. Capitanescu is with the Interdisciplinary Centre for Security, Reliability and Trust (SnT), University of Luxembourg, L-1359 Luxembourg City, Luxembourg (e-mail: florin.capitanescu@uni.lu).

L. Wehenkel is with the Department of Electrical Engineering and Computer Science, University of Liège, B4000 Liège, Belgium (e-mail: 1.wehenkel@ulg.ac.be).

Digital Object Identifier 10.1109/TPWRS.2012.2220384

\section{B. Optimization Variables}

s Uncertain bus active/reactive power injections.

$\mathbf{x}_{0} \quad$ State variables (i.e., magnitude and angle of voltages) in pre-contingency state.

$\mathbf{x}_{c} \quad$ State variables after contingency $c$.

$\mathbf{u}_{p} \quad$ Strategic decisions in pre-contingency state.

$\mathbf{u}_{0} \quad$ Preventive controls in pre-contingency state.

$\mathbf{u}_{c} \quad$ Corrective controls after contingency $c$.

$\boldsymbol{\delta} \quad$ Positive relaxations of the post-contingency inequality constraints.

\section{Constants}

$\mathbf{s}^{\min } \quad$ Lower bound on uncertain injections $\mathbf{s}$.

$\mathbf{s}^{\max } \quad$ Upper bound on uncertain injections $\mathbf{s}$.

$\overline{\mathbf{u}}_{0} \quad$ Planned optimal settings of preventive controls.

$\Delta \mathbf{u}_{0} \quad$ Bound on preventive control changes.

$\boldsymbol{\Delta} \mathbf{u}_{c} \quad$ Bound on corrective control changes after contingency $c$.

\section{INTRODUCTION}

D AY-AHEAD operational planning as well as intraday operation of power systems is nowadays affected by increasing levels of uncertainties due to: renewable generation intermittency (mainly wind power and photovoltaics), cross-border interchanges, intraday market coupling, load evolution, etc. [1]. In this context, the traditional deterministic day-ahead operational planning approach (that targets system security for a single forecasted system state in a given period of time of the next day) becomes insufficient.

As renewable generation (e.g., wind power) cannot be predicted with acceptable accuracy 24 to few hours ahead of operation [1], operators have to cope with these uncertainties in one way or another. In this context, a possible approach (not relying on probabilistic models) consists in checking whether, given some range of uncertainties (e.g., defined as intervals on bus active/reactive power injections), the worst-case with respect to each contingency is still controllable by appropriate combinations of preventive and corrective actions. To tackle this problem [2] sets-up a broader framework in the form of a threestage decision making process under uncertainty including slow strategic controls that need to be committed several hours ahead 
in time (e.g., starting up a power plant, postponing maintenance works), fast preventive controls that may be launched in realtime operation (e.g., generation rescheduling) and corrective (or emergency) controls can still be taken in the post-contingency state (e.g., generation rescheduling, network switching, phase shifter actions, etc.). The computation of worst-case scenarios is an essential task of this approach. The worst-cases that cannot be covered by preventive/corrective controls require strategic actions that can be computed using the approach presented in [3].

The worst-case operating conditions of a power system under operational uncertainty have been tackled in the literature mostly in the framework of security margins [4]-[7]. These approaches look for computing minimum security margins under operational uncertainty with respect to either thermal overloads [5], [7] or voltage instability [4], [6], [7]. These approaches yield min-max optimization problems since a security margin is by definition the maximum value of the loading parameter for a given path of system evolution. However these works do not consider the help of preventive or corrective actions to manage the worst operating states.

In [2] the worst-case with respect to a contingency is formulated as a bilevel (min-max) optimization problem which focuses on thermal overload only and uses a DC load flow approximation, and can thus be transformed into a MILP problem for which suitable solvers are available.

Reference [8] tackles a slightly different bilevel worst-case problem that relies on the nonlinear AC network model. It proposes an algorithm to provide an approximate solution of this bilevel problem that relies on the identification of the constraints that are violated by worst uncertainty patterns.

In the present paper we revisit the AC approach of [8] and assess it in the context of benchmark systems of small to very large size, while focusing on thermal overload problems.

Bilevel programming (BP) is a class of NP-hard optimization problems which has received significant attention especially in the context of linear problems, due to both the appealing mathematical properties of the latter and the challenges to devise powerful generic algorithms for combinatorial nonlinear problems [9]. Consequently, in the power systems area, except of few very recent works [10]-[12] mostly linear approximations of nonlinear BP optimization problems have been reported [2], [5], [13]-[17]. The survey paper [9] mentions several generic approaches for BP such as: implicit enumeration (e.g., branch-and-bound), complementary pivoting algorithms, penalty function methods, steepest descent methods, and mathematical programming with equilibrium constraints (MPEC), among others.

The linear BP has been applied to a large variety of problems, e.g., worst overloads [2], [5], terrorist threats [13], [14], worst-case interdictions [15], vulnerability analysis under multiple contingencies [16], contingency-constrained unit commitment [17], etc. Most of these approaches reformulate the lower-level problem by means of Karush-Kuhn-Tucker optimality conditions, leading to a MPEC single level optimization, and further transform the problem into a more tractable MILP, except for [16] which uses results from duality theory and [17] which uses robust optimization techniques.
The nonlinear BP approaches generally attempt to exploit the particular structure of a problem [9], [10]. Reference [10] proposes an approximation of BP that avoids nonlinear constraints and reduces the problem to a combinatorial search. References [11] and [12] use an appealing approach that, following the reasoning of the linear case, transforms the BP into a single level nonlinear MPEC optimization problem. However, this approach is risky as nonlinear MPEC problems can present several difficulties: multiple solutions obtained from different initial points [11], [18] and reliability issues [18].

Our computation of worst-case scenarios under uncertainty also belongs to the class of robust optimization methods [19], [20]. The one-shot robust optimization problems have received little attention so far in power systems, the few existing applications focusing on: network expansion [21], electricity markets [22], and unit commitment [17].

To the authors' knowledge, nowadays there exists no theoretically and practically sound algorithm able to solve in a generic way and reasonably fast our non-convex, nonlinear, and large scale BP problem either by BP methods [9] or by robust optimization methods [20].

In this paper we propose a practical heuristic approach aiming to provide an approximate solution of the original BP problem (which is equivalent to a nonlinear and generally non-convex optimization problem with an infinite number of constraints) by replacing it by a finite number of tractable OPF- or SCOPF-like nonlinear programming problems. Our algorithm is essentially an instantiation of the general method presented in [23]; we refer the interested reader to this paper for a theoretical discussion of the fundamental properties of the approach.

The rest of the paper is organized as follows. Section II provides the general formulation of the robust optimization problem that we want to tackle. Section III presents the proposed algorithms based on ad hoc constraint relaxation techniques. Extensive numerical results with these algorithms and several test systems are provided in Section IV. Section V concludes and discusses directions of further research.

\section{Formulation OF THE PROBLEM}

In the framework of [2], upon which our approach rests, one aims to determine strategic day-ahead decisions $\mathbf{u}_{p}$ such that for each scenario $\mathbf{s}$ that may show up the next day there exists a combination of preventive controls $\mathbf{u}_{0}(\mathbf{s})$ and of corrective (post-contingency) controls $\mathbf{u}_{c}(\mathbf{s}, c)$ leading to an acceptable performance for any contingency $c \in \mathcal{K}$. This problem may be solved by (an iteration over) the following two steps:

1) In day-ahead operation planning, determine for each contingency $c \in \mathcal{K}$ the worst-case operating scenario, considering optimal use of preventive/corrective actions in the next day.

2) Determine a strategic decision $\mathbf{u}_{p}$ to relieve all the constraints violated for all the worst-case scenarios for which no effective combination of next-day preventive and corrective actions was found.

An approach to deal with step 2 was already presented in [3]. In this work we focus on step 1.

The determination of the worst-case operating scenario for a contingency requires defining a "severity" measure to quantify 
operating conditions. A natural choice is to express this severity in terms of the maximum total amount of post-contingency constraints violation. We use an $L_{1}$ norm of branch overloads although the formulation can be adapted to other norms, e.g., $L_{2}$ or $L_{\infty}$, if this is deemed more appropriate in a particular context (e.g., using a $L_{\infty}$ norm would focus on system weak points, i.e., on the most strongly violated constraint).

We define the worst-case scenario for a given contingency $c$ as the operating scenario within a postulated uncertainty interval leading to the largest total overload of post-contingency constraints in the presence of the best possible combination of preventive and corrective actions. Its computation can be done by solving the following BP:

$$
\begin{array}{ll}
\max _{\mathbf{s}^{\min } \leq \mathbf{s} \leq \mathbf{s}^{\max }} \min _{\mathbf{u}_{0}, \mathbf{u}_{c}, \mathbf{x}_{0}, \mathbf{x}_{c}, \boldsymbol{\delta}} & \mathbf{1}^{T} \boldsymbol{\delta} \\
\text { subject to : }: & \mathbf{g}_{0}\left(\mathbf{x}_{0}, \mathbf{u}_{0}, \mathbf{s}\right)=\mathbf{0} \\
& \mathbf{h}_{0}\left(\mathbf{x}_{0}, \mathbf{u}_{0}, \mathbf{s}\right) \leq \mathbf{0} \\
& \mathbf{g}_{c}\left(\mathbf{x}_{c}, \mathbf{u}_{0}, \mathbf{u}_{c}, \mathbf{s}\right)=\mathbf{0} \\
& \mathbf{h}_{c}\left(\mathbf{x}_{c}, \mathbf{u}_{0}, \mathbf{u}_{c}, \mathbf{s}\right) \leq \boldsymbol{\delta} \\
& \left|\mathbf{u}_{0}-\overline{\mathbf{u}}_{0}\right| \leq \Delta \mathbf{u}_{0} \\
& \left|\mathbf{u}_{c}-\mathbf{u}_{0}\right| \leq \Delta \mathbf{u}_{c} \\
& \boldsymbol{\delta} \geq \mathbf{0}
\end{array}
$$

where subscript 0 (resp. $c$ ) refers to the base case or pre-contingency (resp. post-contingency) state, $\mathbf{u}_{0}$ is the vector of preventive actions (e.g., generators active power, phase shifter angle, shunt reactive power injection, transformer ratio, etc.), $\overline{\mathbf{u}}_{0}$ is the vector of planned optimal of base case controls (e.g., obtained previously by a SCOPF which satisfies all contingency constraints relative to the most likely operating scenario forecasted for the considered period of time of the next day), $\mathbf{u}_{c}$ is the vector of corrective actions (e.g., generators active power, phase shifter angle, network switching, etc.), and constraints (6)-(7) aim at avoiding unrealistic preventive and corrective actions. Strategic control actions $\mathbf{u}_{p}$ have not been made explicit, since they are frozen in our context.

The solution of this BP can be interpreted as follows. For each possible value of the operating uncertainty vector $\mathbf{s}$ lying in its domain $\left[\mathbf{s}^{\min } ; \mathbf{s}^{\max }\right]$, the slave SCOPF problem (1)-(8) which includes only one contingency, called hereafter SCOPF-1C, is solved. If its optimal solution, i.e., the minimum overall violation of constraints (5), is equal to zero, it means that the uncertainty pattern does not lead to any constraint violation provided that adequate preventive and/or corrective actions are available. After considering all the values of $\mathbf{s} \in\left[\mathbf{s}^{\min } ; \mathbf{s}^{\max }\right]$, the worstcase scenario $\mathbf{s}_{c}^{\star}$ is the one leading to the largest overall violation of post-contingency constraints.

If the optimal value of this BP problem is strictly positive it means that strategic actions $\mathbf{u}_{p}$ would be required so as to enhance the system controllability during the next day [3]. Otherwise, the considered contingency is manageable by the sole combination of preventive and corrective controls applied during the next day. In this case the formulation looks only for the existence of a feasible set of preventive and corrective actions for each scenario and contingency rather than for their optimal values. We note also that the worst-case scenario may change according to the considered contingency $c$, and with the range of preventive/corrective control actions that are allowed, which in turn will depend on the choice of $\mathbf{u}_{p}$.

\section{COMPUTATION OF THE WORST UNCERTAINTY PATTERN FOR A Single CONTINGENCY}

\section{A. Principle and Assumptions}

We propose a practical heuristic approach aiming to provide an acceptable solution of the original BP by decomposing it into a number of OPF- or SCOPF-like problems.

To explain the approach, let us first assume that the preventive and corrective actions are frozen. In this particular case the general BP (1)-(8) reduces to

$$
\begin{aligned}
\max _{\mathbf{s}^{\min } \leq \mathbf{s} \leq \mathbf{s}^{\max }} \min _{\mathbf{x}_{0}, \mathbf{x}_{c}, \boldsymbol{\delta}} & \mathbf{1}^{T} \boldsymbol{\delta} \\
\text { subject to : } & \mathbf{g}_{0}\left(\mathbf{x}_{0}, \mathbf{s}\right)=\mathbf{0} \\
& \mathbf{h}_{0}\left(\mathbf{x}_{0}, \mathbf{s}\right) \leq \mathbf{0} \\
& \mathbf{g}_{c}\left(\mathbf{x}_{c}, \mathbf{s}\right)=\mathbf{0} \\
& \mathbf{h}_{c}\left(\mathbf{x}_{c}, \mathbf{s}\right) \leq \boldsymbol{\delta} \\
& \boldsymbol{\delta} \geq \mathbf{0}
\end{aligned}
$$

where the dependencies on the frozen values of $\mathbf{u}_{0}$ and $\mathbf{u}_{c}$ are hidden.

Observe that, as preventive/corrective actions are frozen, the role of the relaxation variable $\delta$ becomes passive, i.e., it only measures the amount of violations of (13) at the solution of the post-contingency load flow (12) for a given uncertainty s. Thus the upper level variable $\mathbf{s}$ becomes the single control variable of the bilevel problem. Hence this bilevel problem looks for the value of $\mathbf{s} \in\left[\mathbf{s}^{\min } ; \mathbf{s}^{\max }\right]$ that maximizes the overall violation of post-contingency constraints (13). Furthermore, if we assume that the frozen values of $\mathbf{u}_{0}$ and $\mathbf{u}_{c}$ correspond to the optimal solution of the original BP (1)-(8), and if we suppose that we know beforehand the subset $\mathcal{V C}$ of constraints among $\mathbf{h}_{c}\left(\mathbf{x}_{c}, \mathbf{s}\right) \leq \mathbf{0}$ that are violated at this solution, then the bilevel problem can be expressed as an equivalent single level optimization problem. Consequently, one can compute the worst uncertainty pattern and its corresponding maximum degree of constraints violation by solving the following SCOPF-1C problem (see the Appendix for a detailed formulation of this problem):

$$
\begin{aligned}
\mathbf{s}_{c}^{\star}=\arg \max _{\mathbf{x}_{0}, \mathbf{x}_{c}, \mathbf{s}} & \sum_{j \in \mathcal{V} \mathcal{C}} h_{c j}\left(\mathbf{x}_{c}, \mathbf{s}\right) \\
\text { s.t. } & \mathbf{s}^{\min } \leq \mathbf{s} \leq \mathbf{s}^{\max } \\
& \mathbf{g}_{0}\left(\mathbf{x}_{0}, \mathbf{s}\right)=\mathbf{0} \\
& \mathbf{h}_{0}\left(\mathbf{x}_{0}, \mathbf{s}\right) \leq \mathbf{0} \\
& \mathbf{g}_{c}\left(\mathbf{x}_{c}, \mathbf{s}\right)=\mathbf{0}
\end{aligned}
$$

where the inequality constraints which do not belong to the set $\mathcal{V C}$ have been removed beforehand from the SCOPF-1C problem because they are supposed to be known a priori as being anyway satisfied [by definition of the set $\mathcal{V C}$, i.e., $h_{c j}\left(\mathbf{x}_{c}, \mathbf{s}\right) \leq$ $0, \forall j \notin \mathcal{V C}]$.

The proposed approach therefore computes the worst uncertainty pattern by identifying in a combinatorial fashion the set $\mathcal{V C}$. To this end we identify the set $\mathcal{A P C}$ of all possible sets 
of problematic constraints, where a set $\mathcal{P C}$ of problematic constraints comprises post-contingency constraints for which there exists an uncertainty pattern leading to their simultaneous violation in the absence of any preventive/corrective action. Each set $\mathcal{P C}$ has associated a worst uncertainty pattern, i.e., a pattern that leads to the largest total violation of all the constraints of this set, which we call problematic pattern. We denote with $\mathcal{P} \mathcal{P}$ the set of problematic patterns corresponding to all possible sets of problematic constraints $\mathcal{A P C}$.

The complete algorithm comprises three main steps that are described hereafter in Sections III-B, III-C, and III-D.

\section{B. Determination of the Set of Problematic Patterns Without Any Preventive/Corrective Action}

The proposed algorithm is as follows:

0) Initialization: $\mathcal{A P C}=\emptyset$, and $\mathcal{P} \mathcal{P}=\emptyset$.

1) For each inequality constraint $j=1, \ldots, n_{h}$, where $n_{h}$ is the size of vector $\mathbf{h}_{c}$, compute its corresponding worst uncertainty pattern (i.e., that maximizes the violation of post-contingency constraint $j$ ) by solving the following SCOPF-1C problem:

$$
\begin{aligned}
\mathbf{s}_{c j}^{\star}=\arg \max _{\mathbf{x}_{0}, \mathbf{x}_{c}, \mathbf{s}} & h_{c j}\left(\mathbf{x}_{c}, \mathbf{s}\right) \\
\text { s.t. } & \mathbf{s}^{\min } \leq \mathbf{s} \leq \mathbf{s}^{\max } \\
& \mathbf{g}_{0}\left(\mathbf{x}_{0}, \mathbf{s}\right)=\mathbf{0} \\
& \mathbf{h}_{0}\left(\mathbf{x}_{0}, \mathbf{s}\right) \leq \mathbf{0} \\
& \mathbf{g}_{c}\left(\mathbf{x}_{c}, \mathbf{s}\right)=\mathbf{0} .
\end{aligned}
$$

If the objective of this optimization problem is less or equal to zero, it means that, whatever the uncertainty pattern, the constraint $j$ is always satisfied. Consequently this constraint may be omitted in the subsequent steps of the algorithm.

Because for the computation of the maximum violation of constraint $j$ the other post-contingency inequality constraints $h_{c i}\left(\mathbf{x}_{c}, \mathbf{s}\right) \leq 0, i \neq j$ have been removed from the optimization problem some of them may be violated at the optimum.

If only constraint $j$ is violated at this SCOPF-1C solution, we augment the set of problematic patterns $\mathcal{P P} \leftarrow \mathcal{P} \mathcal{P} \cup$ $\left\{\mathbf{s}_{c j}^{\star}\right\}$. Otherwise, augment the set $\mathcal{A P C} \leftarrow \mathcal{A P C} \cup \mathcal{P C} \mathcal{C}_{j}$, where the set of problematic constraints $\mathcal{P C}$ is composed by all violated constraints at the SCOPF-1C (20)-(24) solution.

2) Compute the worst uncertainty pattern of each set of problematic constraints $\mathcal{P C} \mathcal{C}_{j} \in \mathcal{A} \mathcal{P C}$ by solving the SCOPF-1C (15)-(19), with set $\mathcal{P C}$ replacing set $\mathcal{V C}$. Let $\mathbf{s}_{c \mathcal{P C}}^{\star}$ denote the worst uncertainty pattern derived from this problem (note that this step is skipped for sets $\mathcal{P} \mathcal{C}_{j}$ that contain a single constraint, since this computation has been already performed in the previous step). Augment the set of problematic uncertainty patterns $\mathcal{P} \mathcal{P} \leftarrow \mathcal{P} \mathcal{P} \cup\left\{\mathbf{s}_{c}^{\star} \mathcal{P} \mathcal{C}_{j}\right\}$. Notice that if, for each and every constraint $j=1, \ldots, n_{h}$, the objective of the SCOPF-1C (20)-(24) is less or equal to zero, then the worst uncertainty pattern for the contingency $c$ does not lead to any post-contingency constraint violation and the overall computation terminates.

\section{Checking Whether Corrective Actions Alone Suffice to Face the Identified Problematic Patterns}

For each problematic scenario $\mathbf{s} \in \mathcal{P} \mathcal{P}$ indentified in the previous step, we check whether corrective actions alone would suffice to remove the violated constraints, by solving the following OPF problem:

$$
\begin{aligned}
\min _{\mathbf{x}_{c}, \mathbf{u}_{c}, \boldsymbol{\delta}} & \mathbf{1}^{T} \boldsymbol{\delta} \\
\text { s.t. } & \mathrm{g}_{c}\left(\mathbf{x}_{c}, \mathbf{u}_{c}, \mathbf{s}\right)=\mathbf{0} \\
& \mathbf{h}_{c}\left(\mathbf{x}_{c}, \mathbf{u}_{c}, \mathbf{s}\right) \leq \boldsymbol{\delta} \\
& \left|\mathbf{u}_{c}-\overline{\mathbf{u}}_{0}\right| \leq \Delta \mathbf{u}_{c} \\
& \boldsymbol{\delta} \geq \mathbf{0} .
\end{aligned}
$$

Observe that this problem does not include base case constraints (22)-(23) since any stress pattern $\mathbf{s}$ computed from the SCOPF-1C (20)-(24) must indeed satisfy these constraints.

\section{Checking Whether Both Preventive/Corrective Actions Suffice to Face the Identified Problematic Patterns}

For each problematic scenario $\mathbf{s} \in \mathcal{P} \mathcal{P}$ for which corrective actions alone do not suffice to solve the problem, we check whether a suitable combination of preventive and corrective actions would be able to meet post-contingency constraints, by solving the following SCOPF-1C problem:

$$
\begin{aligned}
\min _{\mathbf{x}_{0}, \mathbf{x}_{c}, \mathbf{u}_{0}, \mathbf{u}_{c}, \boldsymbol{\delta}} & \mathbf{1}^{T} \boldsymbol{\delta} \\
\text { s.t. } & \mathrm{g}_{0}\left(\mathbf{x}_{0}, \mathbf{u}_{0}, \mathbf{s}\right)=\mathbf{0} \\
& \mathbf{h}_{0}\left(\mathbf{x}_{0}, \mathbf{u}_{0}, \mathbf{s}\right) \leq \mathbf{0} \\
& \mathbf{g}_{c}\left(\mathbf{x}_{c}, \mathbf{u}_{0}, \mathbf{u}_{c}, \mathbf{s}\right)=\mathbf{0} \\
& \mathbf{h}_{c}\left(\mathbf{x}_{c}, \mathbf{u}_{0}, \mathbf{u}_{c}, \mathbf{s}\right) \leq \boldsymbol{\delta} \\
& \left|\mathbf{u}_{0}-\overline{\mathbf{u}}_{0}\right| \leq \Delta \mathbf{u}_{0} \\
& \left|\mathbf{u}_{c}-\mathbf{u}_{0}\right| \leq \Delta \mathbf{u}_{c} \\
& \boldsymbol{\delta} \geq \mathbf{0} .
\end{aligned}
$$

If for at least one uncertainty pattern in $\mathcal{P} \mathcal{P}$ the objective (30) is strictly positive then the best combination of preventive and corrective actions is not able to meet post-contingency constraints and hence strategic preventive actions will be required.

\section{E. Remarks}

The problematic pattern leading to the largest value of the objective (30) approximates the worst uncertainty pattern of the original BP (1)-(8). The degree of approximation of our solution depends on the distance between the worst uncertain patterns computed with and without preventive/corrective actions.

Note that the worst uncertainty pattern of the original BP attempts at maximizing the overall violation of a set of constraints while exploiting weaknesses in available preventive/corrective actions. Fortunately, since all sets of violated constraints are enumerated in step 1, the algorithm can identify tricky situations where patterns leading to smaller constraint violations than the worst pattern in the absence of control actions may turn out to be more dangerous in the presence of control actions due to the lack of efficient control actions. As this enumeration reduces the effect of inefficient control actions then, as both optimizations, 


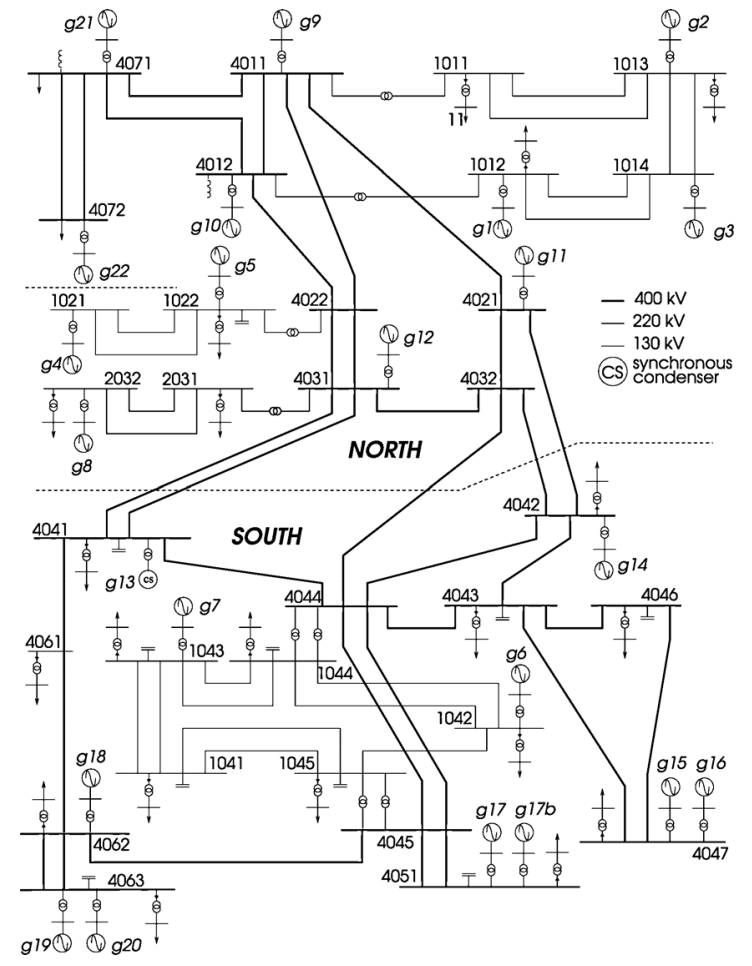

Fig. 1. Modified Nordic32 test system.

the original BP and our heuristic, tend to maximize violated constraints, we expect that the approximate solution computed in this way is reasonably close to the real one.

\section{NumericAl RESUlts}

\section{A. Description of the Test Systems}

We consider three test systems denoted by A, B, and C. System A is a variant of the "Nordic 32" system [26] (see Fig. 1). System B is a modified planning model of the RTE 1 (the French TSO) system. System C is a modified model of the interconnected EHV European power system which spans from Portugal and Spain to Ukraine, Russia and Greece. Notice that in this model the real parameters of the individual power systems components (e.g., lines, transformers, etc.), the network topology, as well as the limits on: generators active/reactive powers, transformers ratio and angle, voltages, and branch currents have been biased. Nevertheless, this model is representative for the European interconnection in terms of system size and complexity.

A summary of the characteristics of our test systems is given in Table I, where $n, g, c, l, t, o, a, s, u$, the number of: buses, generators, loads, lines, all transformers, transformers with controllable ratio, phase shifters, shunt elements, and uncertain active/reactive injections, respectively.

\section{B. Simulation Assumptions}

Uncertainty consists in variable active and reactive power injections at relevant buses (e.g., large loads, border nodes) modeled by constraints (39)-(40). Furthermore, the total variation of

\footnotetext{
${ }^{1}$ Note that our computations do not necessarily represent the current or past operational practice in RTE.
}

TABLE I

TEST Systems CHARACTERISTICS

\begin{tabular}{|c||c|c|c|c|c|c|c|c|c|}
\hline system & $n$ & $g$ & $c$ & $l$ & $t$ & $o$ & $a$ & $s$ & $u$ \\
\hline A & 60 & 23 & 22 & 57 & 27 & 4 & 0 & 14 & 44 \\
\hline B & 1203 & 177 & 767 & 1394 & 403 & 0 & 0 & 11 & 490 \\
\hline C & 8387 & 1865 & 4669 & 12474 & 2087 & 589 & 84 & 178 & 1595 \\
\hline
\end{tabular}

uncertain active (resp. reactive) power injections, modeled by constraints (41)-(42), is trimmed to the range $\pm 100 \mathrm{MW}$ (resp. MVar).

The following simulation cases are considered:

- case 0: the contingency is simulated at the classical SCOPF solution by a power flow program (hence without considering any corrective action);

- case WP: the worst uncertainty pattern (WP) corresponding to the contingency, computed by solving the SCOPF-1C (15)-(19);

- case WP + CA: the worst uncertainty pattern corresponding to the contingency considering corrective actions (CA), computed by solving the OPF (25)-(29);

- case WP + PA + CA: the worst uncertainty pattern corresponding to the contingency considering both preventive actions (PA) and corrective actions, computed by solving the SCOPF-1C (30)-(37).

As preventive and corrective control actions we only consider active generation power rescheduling, the control ranges and the numbers of generators participating in this rescheduling being properly chosen for each case.

All NLP OPFs and SCOPFs problems are solved using our interior-point based NLP solver described in [24].

\section{Results Using System A}

We first compute a reference schedule for the nominal scenario by minimizing generation cost with a SCOPF formulation [25] including 33 relevant N-1 contingencies. At this SCOPF optimum we compute the worst uncertainty pattern for each contingency.

We illustrate the search procedure of the worst uncertainty pattern, described in Sections III-B, III-C, and III-D, for the loss of line 4011-4021.

At step 1 of the algorithm we notice that, only for 3 lines (4031-4032, 4012-4022, and 4022-4031) considered separately, there exists uncertain patterns leading to overload. In particular the worst pattern with respect to any of these 3 lines also overloads the two other lines (see Table II). Then we build up all sets of problematic constraints as all possible combinations among these 3 lines.

At step 2 we compute the worst pattern for each set of problematic constraints. Table II provides the lines overloaded for the 6 sets of problematic constraints $\mathcal{P C}$. Due to the simplicity of the test network and the small number of lines overloaded, only two problematic patterns (set $\mathcal{P} \mathcal{P}$ ) have been found. For instance the worst pattern for the overload of line 4031-4032 coincides with the worst pattern for any set of problematic constraints $\mathcal{P C}$ which includes line 4031-4032. Also, the worst patterns for the overload of lines 4012-4022 and 4022-4031 coincide, as expected, given the location of these lines (see Fig. 1). 
This article has been accepted for inclusion in a future issue of this journal. Content is final as presented, with the exception of pagination.

TABLE II

LiNES OVERLOADED (\%) AND OVERALL OVERLOAD (\%) FOR All SETS OF PROBlematic CONSTRAints $\mathcal{P} \mathcal{C}$

\begin{tabular}{|c|c|c|c|c|}
\hline \multirow{2}{*}{$\begin{array}{c}\text { all sets } \\
\mathcal{P C}\end{array}$} & \multicolumn{3}{|c|}{ lines overloaded } & $\begin{array}{c}\text { overall } \\
\text { overload }\end{array}$ \\
\cline { 2 - 4 } & $4031-4032$ & $4012-4022$ & $4022-4031$ & 29.2 \\
\hline $4031-4032$ & 19.7 & 7.0 & 2.5 & 23.2 \\
\hline $4012-4022$ & 13.3 & 7.2 & 2.7 & 2.7 \\
\hline $4022-4031$ & 13.3 & 7.2 & 2.7 & 29.2 \\
\hline $\begin{array}{r}4031-4032, \\
4012-4022\end{array}$ & 19.7 & 7.0 & 2.5 & \\
\hline $\begin{array}{r}4031-4032, \\
4022-4031\end{array}$ & 19.7 & 7.0 & 2.5 & 29.2 \\
\hline $\begin{array}{r}4012-4022, \\
4022-4031\end{array}$ & 13.3 & 7.2 & 2.7 & 23.2 \\
\hline $\begin{array}{r}4031-4032, \\
4012-4022, \\
4022-4031\end{array}$ & 19.7 & 7.0 & 2.5 & 29.2 \\
\hline
\end{tabular}

TABLE III

LoAding (\%) OF CRITICAL Lines IN VARIOUS CASES For the Two Problematic PatTerns

\begin{tabular}{|c|c|c|c|c|}
\hline line & 0 & $W P$ & $W P+C A$ & $W P+C A+P A$ \\
\hline \multicolumn{5}{|c|}{ first problematic pattern } \\
\hline $4031-4032$ & $\mathbf{1 0 2 . 4}$ & $\mathbf{1 1 9 . 7}$ & $\mathbf{1 1 6 . 3}$ & $\mathbf{1 0 9 . 3}$ \\
\hline $4012-4022$ & 95.3 & $\mathbf{1 0 7 . 0}$ & $\mathbf{1 0 3 . 7}$ & 99.9 \\
\hline $4022-4031$ & 90.1 & $\mathbf{1 0 2 . 5}$ & 99.4 & 91.1 \\
\hline \multicolumn{6}{|c|}{ second problematic pattern } \\
\hline $4031-4032$ & $\mathbf{1 0 2 . 4}$ & $\mathbf{1 1 3 . 3}$ & $\mathbf{1 0 7 . 2}$ & $\mathbf{1 0 0 . 5}$ \\
\hline $4012-4022$ & 95.3 & $\mathbf{1 0 7 . 2}$ & $\mathbf{1 0 3 . 9}$ & 100.0 \\
\hline $4022-4031$ & 90.1 & $\mathbf{1 0 2 . 7}$ & 99.8 & 92.0 \\
\hline
\end{tabular}

Next we check for the two problematic patterns whether the preventive/corrective actions suffice. We provide, in Table III, the loading of critical lines in various cases (column 0 corresponds to the base case, WP to the worst pattern without control, WP + CA to possible corrective control actions only, and $\mathrm{WP}+\mathrm{CA}+\mathrm{PA}$ corresponds to the best combination of both corrective and preventive control actions).

We conclude that the first problematic pattern is the worst pattern for this contingency as it leads to the largest overall overload in the case WP + CA + PA.

\section{Results Using System B}

During the application of our procedure only three contingencies among the total 1029 postulated ones, denoted hereafter $\mathrm{C} 1$, $\mathrm{C} 2$, and $\mathrm{C} 3$, lead to overloads on their worst-case. Furthermore, there is a single problematic pattern associated to each contingency since only one line can be overloaded given the range of uncertainty.

Table IV provides the overall line overload for these critical contingencies in various cases. Comparing the results obtained for contingencies $\mathrm{C} 1$ and $\mathrm{C} 2$ one can observe that the algorithm is able to identify tricky situations where patterns leading to smaller constraint violations in the absence of control actions are more dangerous in the presence of control actions due to the lack of efficient control actions, as $\mathrm{C} 1$ is worse than $\mathrm{C} 2$ in case WP but $\mathrm{C} 2$ is worse than $\mathrm{C} 1$ in case $\mathrm{WP}+\mathrm{CA}+\mathrm{PA}$.

\section{E. Results Using System C}

Because the base case is already quite constrained (e.g., few lines are loaded at more than 90\%) we limit ourselves the worstcase analysis to the pre-contingency state only. In the absence
TABLE IV

Overall Line OverLoAd (MVA) FOR CRITICAL CONTINGENCIES

\begin{tabular}{|c|c|c|c|}
\hline \multirow{2}{*}{$\begin{array}{c}\text { critical } \\
\text { contingency }\end{array}$} & \multicolumn{3}{|c|}{ case } \\
\cline { 2 - 4 } & 0 & WP & WP+CA+PA \\
\hline C1 & 45 & 466 & 149 \\
\hline C2 & 24 & 658 & 139 \\
\hline C3 & - & 233 & 13 \\
\hline
\end{tabular}

TABLE V

LiNES OVERLOADED (MVA) AND OVERALL OVERLOAD (MVA) FOR ALl SETS OF PROBlematic CONSTRAINTS $\mathcal{P C}$

\begin{tabular}{|c|c|c|c|c|}
\hline all sets & \multicolumn{2}{|c|}{ lines overloaded } & overall \\
\cline { 2 - 4 } $\mathcal{P C}$ & L1 & L2 & L3 & overload \\
\hline L1 & 72.5 & 41.5 & 104.5 & 218.5 \\
\hline L2 & 47.1 & 180.2 & 98.1 & 325.4 \\
\hline L3 & 67.9 & 116.1 & 125.6 & 309.6 \\
\hline L1, L2 & 52.4 & 178.9 & 106.1 & 337.4 \\
\hline L1, L3 & 70.6 & 93.8 & 121.6 & 286.0 \\
\hline L2, L3 & 52.3 & 177.3 & 112.5 & 342.1 \\
\hline L1, L2, L3 & 54.8 & 173.7 & 114.8 & 343.3 \\
\hline
\end{tabular}

of precise information we consider uncertain injections at 1595 loads (the largest ones out of the total of 4669 loads) in the range of $-10 \%$ to $+10 \%$ of the nominal active/reactive load.

Several features of the worst-case problem [e.g., almost all uncertain injections bound constraints (39)-(40) are binding at the optimum, the bounds of many injections are very narrow, and the reactive power limits of a significant number of generators are very tight among others] lead to very challenging worst-case computations. In these conditions we encountered some convergence problems when computing worst patterns. Divergence of IPM manifests as the iterations becomes stuck at a nonoptimal point as one approaches too early the feasibility boundary. In particular the presence of many narrow bounds leads to smaller steps on the Newton direction (so as not to violate positivity constraints of slacks and their corresponding dual variables) and therefore to slow convergence. These issues call for the use of very robust NLP solvers as well as to a realistic definition of bounds of uncertain injections.

To avoid these reliability problems we relaxed our assumptions in two ways: we consider as uncertain only active power injections and we relaxed to some extent the too tight reactive power limits of generators (they can be alternatively handled as generators with constant reactive power output). The former assumption is reasonable as the main source of uncertainty stems from the active power of intermittent renewable generation.

Table V provides the lines overloaded for the 6 sets of problematic constraints $\mathcal{P C}$. One can notice that in this very meshed system and with a large number of degrees of freedom for uncertain injections the worst pattern changes with the set of problematic constraints.

Compared to the previous two systems the case WP + CA which consists in solving the OPF (25)-(29) in order to alleviate overloads in the worst-cases takes further advantage of the presence of phase shifters as corrective actions. The latter suffice in most cases to remove the overloads. Where this is not the case the joint optimization of phase shifters and generators active power remove all overloads.

The visualization of worst uncertain flows patterns constitutes a very important piece of information for a TSO. Figs. 2 


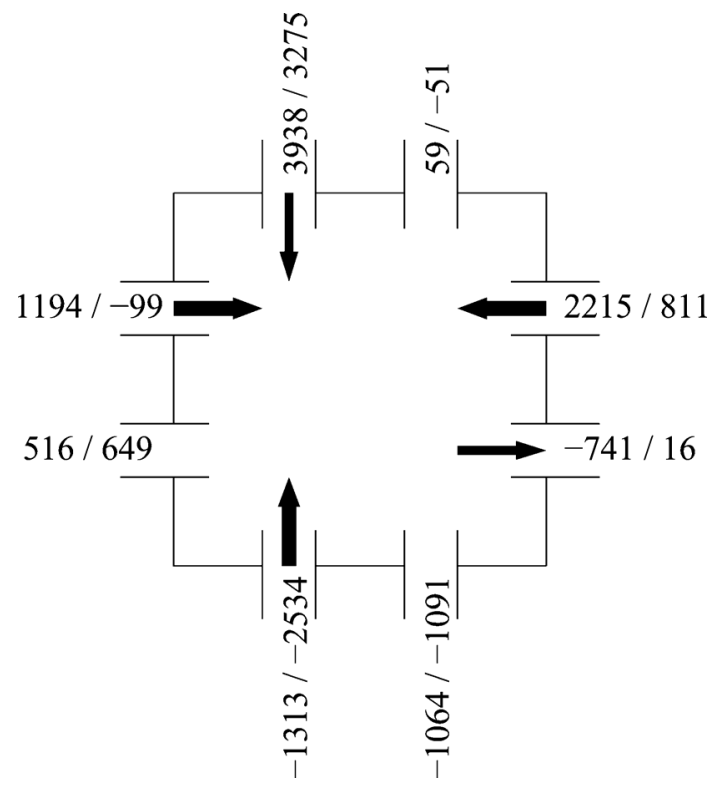

Fig. 2. Active power flow export (MW) for a sub-system in both base case and worst-case of line L1.

and 3 propose a simple top layer situation awareness visualization $^{2}$ of the active power flow export (MW) for a sub-system by a region in both base case and the worst-cases with respect to line L1, and lines L1, L2, and L3, respectively (see Table V). The arrows in the figures indicate the main shift in power flow exchanges with the neighboring TSOs, the width of the arrow being proportional to the flow shift. As expected depending on the set of problematic constraints considered the flow patterns may be very different. Observe that significant changes in the export/import occur in the worst-case compared to the base case and that these changes are more important in the worst-case of the three lines which means that the latter is more sensitive to the external uncertainties of the sub-system considered.

\section{F. Computational Issues}

We provide in Table VI the computing times (on a computer with 1.9-GHz, 2-GB RAM) needed to determine the worst-case with respect to a contingency for a given set of violated constraints.

The proposed algorithm is computationally intensive and depends on the total number of inequality constraints $n_{h}$, the size of the set $\mathcal{P} \mathcal{P}$, and the number of postulated contingencies (the size of set $\mathcal{K}$ ). To reduce its computational time three solutions can be envisaged:

- use parallel computations for the various SCOPF-1C problems;

- the solution of OPF (25)-(29) can be skipped, since it is performed for the sake of distinguishing between cases where corrective actions alone suffice or not to satisfy worst-case constraints, and replaced with the solution of SCOPF (30)-(37);

${ }^{2}$ More detailed layers of visualization are possible for cases of interest. For the sake of simplicity we show only one sub-system but the picture can be extended to neighbors sub-systems in order to ascertain whether there exists a certain trend in the power flows stemming from more far uncertainties.

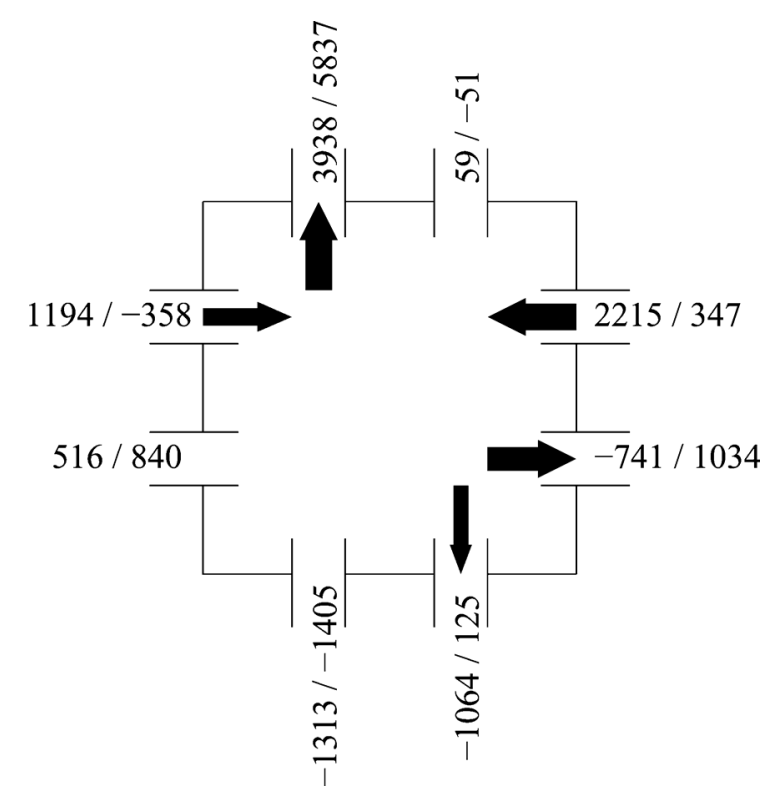

Fig. 3. Active power flow export (MW) for a sub-system in both base case and worst-case of lines L1, L2, and L3.

TABLE VI

RANGE OF CPU TIMES FOR THE WORST-CASE COMPUTATION

\begin{tabular}{|c|c|}
\hline system & time $(\mathrm{s})$ \\
\hline $\mathrm{A}$ & $0.2 \ldots 0.3$ \\
\hline $\mathrm{B}$ & $2.2 \ldots 5.1$ \\
\hline $\mathrm{C}$ & $46.5 \ldots 97.1$ \\
\hline
\end{tabular}

- not all inequalities $\mathbf{h}_{c}\left(\mathbf{x}_{c}, \mathbf{s}\right) \leq \mathbf{0}$ should be treated but only those that are closer to their limits and hence prone to be violated (i.e., the weak-points). TSO expertise can be very useful to filter-out harmless constraints and reduce the set of postulated contingencies $\mathcal{K}$.

\section{CONCLUSION AND Future WORKS}

This paper has proposed a heuristic approach to compute the worst-case under operation uncertainty for a contingency with respect to overloads and in particular to check whether there exist appropriate combinations of preventive and corrective actions to face this worst-case.

The method is intended as a subroutine that may be used in order to reveal the dangerous combinations of uncertainties and contingencies for which the best combination of preventive and corrective actions do not suffice to remove post-contingency overloads. This is a precious information for the system operator, who could wish to take in some optimal manner (taking into account that the worst-case scenarios relate to intrinsically conservative operating conditions) appropriate actions to enhance system security against these worst-case scenarios.

This approach provides as a by-product a very useful information for the static security assessment and control under uncertainty as postulated contingencies can be classified into four clusters according to the type of actions needed to cover the worst-case: 1) contingencies which do not require preventive or corrective actions, 2) contingencies which require only corrective actions, 3) contingencies which require corrective and preventive actions, and 4) contingencies for which the security 
of the system cannot be ensured even by the best combination of available preventive and corrective actions.

The approach computes in a tractable way an approximate solution of the intractable benchmark non-convex nonlinear bilevel worst-case optimization problem, by decomposing it into a finite number of tractable OPF-like and SCOPF-like optimization problems which are solved sequentially.

Although the approach is computationally intensive, the computing times obtained in our simulations suggest already that the approach is practically usable, by resorting to appropriate massively parallel computation architectures in the context of day-ahead operation planning and for medium size systems. On the other hand the approach might not meet more stringent computational requirements in the context of very large-scale systems. In this case, simplified linear approaches as the one proposed in [2] could be used instead, at the price of further approximations.

Future work will be devoted to develop more sophisticated uncertainty models addressing in particular the correlations between exogenous uncertainties as well as to extend the approach to integrate challenging discrete corrective actions (e.g., network switching).

The extension of this approach to under- and over-voltage problems requires further thought. Firstly, large amounts of uncertain intermittent generation have generally a wider impact for thermal overloads than for voltage problems, which are much more local. Secondly, the problem of worst-case with respect to under-voltages may be not well posed. Indeed, as the optimization process would attempt to find the lowest voltage at a bus (or at a pair of buses) under uncertainty, the degree of freedom in uncertain injections may lead to solution degeneracy (i.e., cases where there are an infinite number of worst-cases because the system reaches its loadability limit only due to few efficient injections, whereas other injections are free to vary but without being able to worsen this limit as the load flow equations will have no solution). On the other hand, reaching the loadability limit could prevent efficient uncertain injections to further degradate the state of the system. In addition, the proper computation of worst-cases with respect to voltage instability essentially precludes the use of static tools, and should instead rely on dynamic models [7].

\section{APPENDIX}

The compact SCOPF-1C formulation (15)-(19) can be detailed as follows:

$$
\begin{array}{ll}
\max & \sum_{i j \in \mathcal{V} \mathcal{C}} I_{i j}^{c}\left(V_{i}^{c}, V_{j}^{c}, \theta_{i}^{c}, \theta_{j}^{c}\right) \\
\text { s.t. } \quad & P_{u i}^{\min } \leq P_{u i} \leq P_{u i}^{\max }, \quad \forall i \in \mathcal{N} \\
& Q_{u i}^{\min } \leq Q_{u i} \leq Q_{u i}^{\max }, \quad \forall i \in \mathcal{N} \\
& P_{u}^{\min } \leq \sum_{i \in \mathcal{N}} c_{P i} P_{u i} \leq P_{u}^{\max } \\
& Q_{u}^{\min } \leq \sum_{i \in \mathcal{N}} c_{Q i} Q_{u i} \leq Q_{u}^{\max } \\
P_{g i}^{0}-P_{l i}+c_{P i} P_{u i} & -\sum_{j \in \mathcal{B}_{i}^{0}} P_{i j}^{0}\left(V_{i}^{0}, V_{j}^{0}, \theta_{i}^{0}, \theta_{j}^{0}\right)=0, \quad \forall i \in \mathcal{N}(
\end{array}
$$

$$
\begin{aligned}
& Q_{g i}^{0}-Q_{l i}+c_{Q i} Q_{u i} \\
& \quad-\sum_{j \in \mathcal{B}_{i}^{0}} Q_{i j}^{0}\left(V_{i}^{0}, V_{j}^{0}, \theta_{i}^{0}, \theta_{j}^{0}\right)=0, \forall i \in \mathcal{N} \\
& Q_{g i}^{\min } \leq Q_{g i}^{0} \leq Q_{g i}^{\max }, \quad \forall i \in \mathcal{G} \\
& I_{i j}^{0}\left(V_{i}^{0}, V_{j}^{0}, \theta_{i}^{0}, \theta_{j}^{0}\right) \leq I_{i j}^{\max 0}, \quad \forall i, j \in \mathcal{N} \\
& V_{i}^{\min 0} \leq V_{i}^{0} \leq V_{i}^{\max 0}, \quad \forall i \in \mathcal{N} \backslash \mathcal{G} \\
& P_{g i}^{c}-P_{l i}+c_{P i} P_{u i} \\
& \quad-\sum_{j \in \mathcal{B}_{i}^{c}} P_{i j}^{c}\left(V_{i}^{c}, V_{j}^{c}, \theta_{i}^{c}, \theta_{j}^{c}\right)=0, \quad \forall i \in \mathcal{N}( \\
& Q_{g i}^{c}-Q_{l i}+c_{Q i} Q_{u i} \\
& \quad-\sum_{j \in \mathcal{B}_{i}^{c}} Q_{i j}^{c}\left(V_{i}^{c}, V_{j}^{c}, \theta_{i}^{c}, \theta_{j}^{c}\right)=0, \quad \forall i \in \mathcal{N}(49) \\
& Q_{g i}^{\min } \leq Q_{g i}^{c} \leq Q_{g i}^{\max }, \quad \forall i \in \mathcal{G} \\
& V_{i}^{\min c} \leq V_{i}^{c} \leq V_{i}^{\max c}, \quad \forall i \in \mathcal{N} \backslash \mathcal{G}
\end{aligned}
$$

where superscript 0 (resp. $c$ ) refers to the base case (resp. contingency $c$ state), objective (38) refers to overloads, $P_{u i}$ (resp. $Q_{u i}$ ) denotes uncertain active (resp. reactive) power injection at bus $i, c_{P i}, c_{Q i} \in\{0,1\}$ are coefficients indicating buses where power injections are uncertain (i.e., $c_{P i}=1$ or $c_{Q i}=1$ ), $\mathcal{N}$ is the set of buses, $\mathcal{G}$ is the set of generators, $\mathcal{B}_{i}$ is the set of branches connected to bus $i$, the other notations being self-explanatory. A slack generator, not shown explicitly in this formulation, is chosen to clear the mismatch due to uncertain injections. Uncertain injections are limited at each individual bus by constraints (39) and (40) as well as overall by constraints (41) and (42). The optimization variables of this problem are: $\left(P_{u i}, Q_{u i}, \theta_{i}^{0}, \theta_{i}^{c}\right), \forall i \in \mathcal{N}$, and $\left(V_{i}^{0}, V_{i}^{c}\right), \forall i \in \mathcal{N} \backslash \mathcal{G}$.

Note that since the base case constraints (43) and (47) are generally less restrictive than contingency constraints, they are also satisfied for the worst contingency pattern, which allows further simplification of this formulation.

\section{ACKNOWLEDGMENT}

The authors would like to thank RTE France (The French TSO), and in particular S. Fliscounakis and P. Panciatici, for their support and valuable comments on the paper. They also acknowledge the support of the European FP7 project PEGASE for their earlier research on the topics developed in this paper.

\section{REFERENCES}

[1] K. De Vos and J. Driesen, "Balancing management mechanisms for intermittent power sources-A case study for wind power in Belgium," in Proc. 6th Int. Conf. European Energy Market, 2009.

[2] P. Panciatici, Y. Hassaine, S. Fliscounakis, L. Platbrood, M. OrtegaVazquez, J. L. Martinez-Ramos, and L. Wehenkel, "Security management under uncertainty: From day-ahead planning to intraday operation," in Proc. IREP Symp., Buzios, Brazil, 2010.

[3] F. Capitanescu, S. Fliscounakis, P. Panciatici, and L. Wehenkel, "Cautious operation planning under uncertainty," IEEE Trans. Power Syst., to be published.

[4] J. Jarjis and F. D. Galiana, "Quantitative analysis of steady state stability in power networks," IEEE Trans. Power App. Syst., vol. PAS100, no. 1, pp. 318-326, 1981.

[5] D. Gan, X. Luo, D. V. Bourcier, and R. J. Thomas, "Min-max transfer capability of transmission interfaces," Int. J. Elect. Power Energy Syst., vol. 25 , no. 5, pp. 347-353, 2003.

[6] I. Dobson and L. Lu, "New methods for computing a closest saddle node bifurcation and worst case load power margin for voltage collapse," IEEE Trans. Power Syst., vol. 8, no. 2, pp. 905-911, May 1993. 
[7] F. Capitanescu and T. Van Cutsem, "Evaluating bounds on voltage and thermal security margins under power transfer uncertainty," in Proc. PSCC Conf., Seville, Spain, Jun. 2002.

[8] F. Capitanescu, S. Fliscounakis, P. Panciatici, and L. Wehenkel, "Dayahead security assessment under uncertainty relying on the combination of preventive and corrective controls to face worst-case scenarios," in Proc. PSCC Conf., Stockholm, Sweden, Aug. 2011.

[9] B. Colson, P. Marcotte, and G. Savard, "An overview of bilevel optimization," Ann. Oper. Res., vol. 1, pp. 235-256, 2007.

[10] A. Pinar, J. Meza, V. Donde, and B. Lesieutre, "Optimization strategies for the vulnerability analysis of the electric power grid," SIAM J. Optimiz., vol. 20, no. 4, pp. 1786-1810, 2010.

[11] K. C. Almeida and F. S. Senna, "Optimal active-reactive power dispatch under competition via bilevel programming," IEEE Trans. Power Syst., vol. 26, no. 4, pp. 2345-2354, Nov. 2011.

[12] H. Haghighat and S. W. Kennedy, "A bilevel approach to operational decision making of a distribution company in competitive environments," IEEE Trans. Power Syst., to be published.

[13] J. M. Arroyo and F. D. Galiana, "On the solution of the bilevel programming formulation of the terrorist threat problem," IEEE Trans. Power Syst., vol. 20, no. 2, pp. 789-797, May 2005.

[14] A. L. Motto, J. M. Arroyo, and F. D. Galiana, "A mixed-integer LP procedure for the analysis of electric grid security under disruptive threat," IEEE Trans. Power Syst., vol. 20, no. 3, pp. 1357-1365, Aug. 2005.

[15] J. Salmeron, K. Wood, and R. Baldick, "Worst-case interdiction analysis of large-scale electric power grids," IEEE Trans. Power Syst., vol. 24, no. 1, pp. 96-104, Feb. 2009.

[16] J. M. Arroyo, "Bilevel programming applied to power system vulnerability analysis under multiple contingencies," IET Gener. Transm. Distrib., vol. 4, no. 2, pp. 178-190, 2010.

[17] A. Street, F. Oliveira, and J. M. Arroyo, "Contingency-constrained unit commitment with $\mathrm{n}-\mathrm{K}$ security criterion: A robust optimization approach," IEEE Trans. Power Syst., vol. 26, no. 3, pp. 1581-1590, Aug. 2011.

[18] L. Platbrood, S. Fliscounakis, F. Capitanescu, P. Panciatici, C. Merckx, and M. Ortega-Vazquez, "Deliverable D3.2: Development of prototype software for system steady-state optimization of the European transmission system," PEGASE Project, 2011. [Online]. Available: http:// www.fp7-pegase.eu/.

[19] A. Ben-Tal and A. Nemirovski, "Robust optimization-Methodology and applications," Math. Program., vol. 92, no. 3, pp. 453-480, May 2002.
[20] D. Bertsimas, D. B. Brown, and C. Caramanis, "Theory and applications of robust optimization," SIAM Rev., vol. 53, no. 3, pp. 464-501, 2011.

[21] S. A. Malcolm and S. A. Zenios, "Robust optimization of power systems capacity expansion under uncertainty," J. Oper. Res. Soc., vol. 45, pp. 1040-1049, 1994.

[22] S. Takriti and S. Ahmed, "On robust optimization of two-stage systems," Math. Program., vol. 99, no. 1, pp. 109-126, 2004.

[23] A. Mutapcic and S. Boyd, "Cutting-Set methods for robust convex optimization with pessimizing oracles," Optimiz. Meth. Softw., vol. 24, no. 3, pp. 381-406, 2009.

[24] F. Capitanescu, M. Glavic, D. Ernst, and L. Wehenkel, "Interior-point based algorithms for the solution of optimal power flow problems," Elect. Power Syst. Res., vol. 77, no. 5-6, pp. 508-517, Apr. 2007.

[25] F. Capitanescu and L. Wehenkel, "A new iterative approach to the corrective security-constrained optimal power flow problem," IEEE Trans. Power Syst., vol. 23, no. 4, pp. 1342-1351, Nov. 2008.

[26] Long-Term Dynamics, Phase II, 1995, Nordic32 system, CIGRE Task Force 38.02.08.

Florin Capitanescu received the degree in electrical power engineering from the University "Politehnica" of Bucharest, Bucharest, Romania, in 1997 and the Ph.D. degree from the University of Liège, Liège, Belgium, in 2003.

His main research interests lie in the field of power systems operation, planning, and control, with particular emphasis on optimization methods and voltage stability. He is currently a research associate with SnT, University of Luxembourg, Luxembourg City, Luxembourg.

Louis Wehenkel received the degree in electrical engineering (electronics) and the Ph.D. degree from the University of Liège Liège, Belgium, in 1986 and 1990 , respectively.

Hee is full Professor of electrical engineering and computer science at the University of Liège. His research interests lie in the fields of stochastic methods for systems and modeling, optimization, machine learning, and data mining, with applications in complex systems, in particular large scale power systems planning, operation and control, industrial process control, bioinformatics, and computer vision. 